Braz J Med Biol Res, October 2011, Volume 44(10) 1048-1053

doi: 10.1590/S0100-879X2011007500117

\section{Effects of chronic corticosterone and imipramine administration on panic and anxiety-related responses}

L. Diniz, B.B. dos Reis, G.M. de Castro, C.C. Medalha and M.B. Viana

The Brazilian Journal of Medical and Biological Research is partially financed by

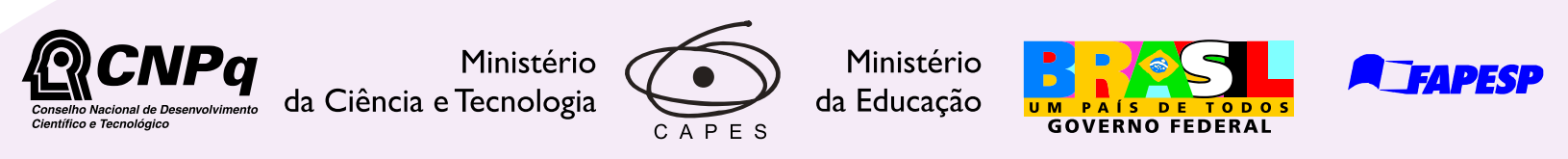

Institutional Sponsors
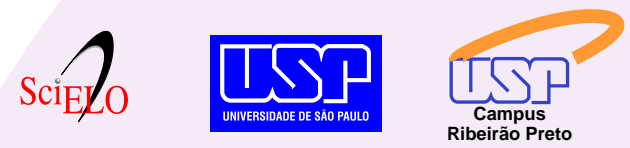

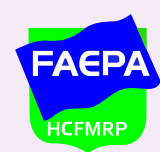

Ф SHIMADZU

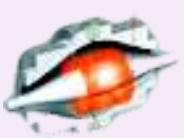

Explore High - Performance MS Orbitrap Technology In Proteomics \& Metabolomics

$\underset{\text { analitica }}{\text { analiticaweb.com.br }}$ SCIENTIFIC 


\title{
Effects of chronic corticosterone and imipramine administration on panic and anxiety-related responses
}

\author{
L. Diniz¹ , B.B. dos Reis², G.M. de Castro², C.C. Medalha² and M.B. Viana² \\ ${ }^{1}$ Departamento de Psiquiatria, Universidade Federal de São Paulo, São Paulo, SP, Brasil \\ ${ }^{2}$ Departamento de Biociências, Universidade Federal de São Paulo, Santos, SP, Brasil
}

\begin{abstract}
It is known that chronic high levels of corticosterone (CORT) enhance aversive responses such as avoidance and contextual freezing. In contrast, chronic CORT does not alter defensive behavior induced by the exposure to a predator odor. Since different defense-related responses have been associated with specific anxiety disorders found in clinical settings, the observation that chronic CORT alters some defensive behaviors but not others might be relevant to the understanding of the neurobiology of anxiety. In the present study, we investigated the effects of chronic CORT administration (through surgical implantation of a 21-day release $200 \mathrm{mg}$ pellet) on avoidance acquisition and escape expression by male Wistar rats (200 $\mathrm{g}$ in weight at the beginning of the experiments, $\mathrm{N}=6-10$ /group) tested in the elevated T-maze (ETM). These defensive behaviors have been associated with generalized anxiety and panic disorder, respectively. Since the tricyclic antidepressant imipramine is successfully used to treat both conditions, the effects of combined treatment with chronic imipramine (15 mg, ip) and CORT were also investigated. Results showed that chronic CORT facilitated avoidance performance, an anxiogenic-like effect $(P<0.05)$, without changing escape responses. Imipramine significantly reversed the anxiogenic effect of CORT $(P<0.05)$, although the drug did not exhibit anxiolytic effects by itself. Confirming previous observations, imipramine inhibited escape responses, a panicolytic-like effect. Unlike chronic CORT, imipramine also decreased locomotor activity in an open field. These data suggest that chronic CORT specifically altered ETM avoidance, a fact that should be relevant to a better understanding of the physiopathology of generalized anxiety and panic disorder.
\end{abstract}

Key words: Corticosterone; Imipramine; Generalized anxiety disorder; Panic disorder; Elevated T-maze

\section{Introduction}

It has been shown that short-term glucocorticoid secretion is essential for the maintenance of several behavioral events, and crucial for the expression of fear/anxiety responses (1-3). In contrast, clinical observations suggest that chronic high levels of glucocorticoids, which are most often a result of prolonged stress, lead to maladaptive anxiety and/or depressive disorders (3).

A number of experimental studies have also demonstrated the anxiogenic effects of the exogenous administration of glucocorticoids. It has been observed, for instance, that post-training injections of corticosterone (CORT) enhance the animals' performance in several aversive tasks, facilitating responses such as inhibitory avoidance and contextual freezing (2). Increases in contextual freezing have also been observed when CORT is administered prior to conditioning (1). On the other hand, chronic CORT does not alter defensive behavior induced by an innate fear stimulus, i.e., exposure to a predator odor (4).

Although the distinction between fear and anxiety has not always been clear, the ethopharmacological analysis of the rodent defensive repertoire developed by Robert and Caroline Blanchard (5) has provided a sound theoretical framework. According to these investigators, anxiety is an emotion related to behavioral inhibition and risk assessment, behaviors that are observed in situations of potential danger, either because the context is new or because the aversive stimulus was once present in the past. On the other hand, fear is associated with behavioral responses that occur in situations of real danger. In such cases, the animal either escapes/flees from the situation or exhibits immobility

Correspondence: M.B. Viana, Departamento de Biociências, Universidade Federal de São Paulo, 11060-001 Santos, SP, Brasil. Fax: +55-11-3868-3203. E-mail: mviana@unifesp.br

Received February 18, 2011. Accepted August 23, 2011. Available online September 9, 2011. Published October 10, 2011. 
(freezing) when there is no possibility of escape. Gray and McNaughton (6) have also emphasized the importance of approach-avoidance conflict for the distinction between anxiety and fear. According to the authors $(6,7)$, behavioral inhibition and risk assessment are responses occurring when an animal is faced with a threat, which involves a conflict between approach and avoidance (e.g., searching for food in an area previously visited by a predator). Risk assessment functions to reduce the level of perceived threat, while behavioral inhibition reduces risk in cases of real threat. On the other hand, when there is no tendency to approach the feared situation, animals often exhibit responses such as escape or flight. These behaviors have been associated with fear $(6,7)$. In terms of psychopathology, it has been proposed that the neurobiological substrates that regulate anxiety are disrupted in generalized anxiety, while dysfunction of the brain circuitry controlling fear has been related to panic disorder $(5,7)$.

In order to better understand how CORT administration alters defensive responses specifically related to anxiety disorders found in clinical settings, in the present study we investigated the effects of chronic CORT (200 mg pellets, 21-day release) on avoidance acquisition and escape expression by male Wistar rats tested in the elevated T-maze (ETM) (8). The pharmacological exploitation of the ETM has shown that these two measurements are differently affected by drug treatments. Thus, compounds representative of three classes of anxiolytics - namely the agonist of benzodiazepine receptors diazepam, the serotonin $1 \mathrm{~A}$ agonist buspirone, and the nonselective type 2 antagonist ritanserin - have been shown to selectively impair ETM avoidance while leaving escape unchanged $(8,9)$. These results are compatible with the view that ETM avoidance relates to generalized anxiety disorder $(8,9)$. In contrast, the escape task is insensitive to different classes of anxiolytics, and is impaired by chronic, but not acute administration of imipramine (10), clomipramine and fluoxetine (11), drugs that are used to treat panic disorder. As a result, ETM escape has been used as an animal model of panic.

Since the tricyclic antidepressant imipramine is successfully used to treat both generalized anxiety and panic disorder, the effects of combined treatment with chronic imipramine (15 mg, ip) and CORT were also investigated. In order to avoid confounding results due to drug effects on locomotor activity, after the tests in the ETM the animals were also evaluated in an open field.

\section{Material and Methods}

\author{
Subjects \\ Thirty-three male Wistar rats (Universidade Federal de \\ São Paulo, CEDEME, Brazil), weighing approximately $200 \mathrm{~g}$ \\ at the beginning of the experiment $(300 \mathrm{~g}$ on the day of the \\ ETM tests), were housed in groups of 5-6 per cage. After \\ surgery to implant the CORT pellets, animals were housed
}

in pairs in Plexiglas-walled cages until testing. Room temperature was controlled $\left(22 \pm 1^{\circ} \mathrm{C}\right)$ and a light-dark cycle was maintained on a 12-h on-off cycle (7:00-19:00 h lights on). Food and water were available throughout the experiments. The present study was approved by the Ethics Committee for Animal Research of the Universidade Federal de São Paulo under protocol number 2179/08 and was performed in compliance with the recommendations of the Brazilian Society of Neuroscience and Behavior (SBNeC), which are based on the US National Institutes of Health Guide for Care and Use of Laboratory Animals.

\section{Apparatus}

The ETM was made of wood and had 3 arms of equal dimensions $(50 \times 12 \mathrm{~cm})$. One of the arms was enclosed by $40-\mathrm{cm}$ high walls and was oriented perpendicularly to two opposed open arms. The whole apparatus was elevated $50 \mathrm{~cm}$ above the floor. To avoid falls, the open arms were surrounded by a 1-cm high Plexiglas rim.

The open field test was performed in a round arena (60 x $60 \mathrm{~cm}$ ), with the floor divided into 16 parts, and walls 50 $\mathrm{cm}$ high. Luminosity at the level of the maze arms or the open field center was 60 lux.

\section{Drugs}

Imipramine (15 mg/kg; Sigma, USA) was dissolved in sterile $0.9 \%$ saline. Control animals received sterile $0.9 \%$ saline alone. Drug and saline were administered ip in a volume of $1 \mathrm{~mL} / \mathrm{kg}$.

\section{Surgery}

Rats were anesthetized with an ip injection of $80 \mathrm{mg} / \mathrm{kg}$ ketamine hydrochloride and $10 \mathrm{mg} / \mathrm{kg}$ xylazine (Agribrands, Brazil). A 1-cm incision was made through the skin of the upper back of the animal and a 2-cm subcutaneous pocket was opened horizontally with a pair of forceps to allow the implant of a slow-release CORT pellet (200 mg, 21-day release; Innovative Research of America, USA). The pellet was inserted into the pocket and the incision sutured. The dose of CORT used here has been reported to result in plasma levels of $13.60 \pm 0.89 \mu \mathrm{g} / \mathrm{dL}$ (mean \pm SEM) in the 18th day of treatment (4). Sham rats were subjected to the same surgical procedure except that a pellet was not implanted. To prevent infection, all animals were injected im with a 0.2-mL pentabiotic preparation (Pentabiótico Veterinário Pequeno Porte; Forte Dodge, Brazil; 600,000 IU benzylpenicillin benzathine, 300,000 IU benzylpenicillin procaine, 300,000 IU benzylpenicillin potassium, $250 \mathrm{mg}$ dihydrostreptomycin sulfate, $250 \mathrm{mg}$ streptomycin sulfate, diluted in $3 \mathrm{~mL}$ sterile $0.9 \%$ saline) and with the antiinflammatory flunixin meglumine $(1 \mathrm{~mL} / \mathrm{kg}, s c$; Banamine; Fort Dodge) at the end of surgery.

\section{Procedure \\ CORT and sham animals were treated daily for 21 days}


with either imipramine $(15 \mathrm{mg} / \mathrm{kg}$, ip) or saline $(1 \mathrm{~mL} / \mathrm{kg})$. On the 20th day, the animals were exposed to one of the open arms of the ETM for $30 \mathrm{~min}$ as described by Sena et al. (12), immediately before treatment. It has been shown that pre-exposure renders the escape task more sensitive to the effects of antipanic drugs because it shortens the latencies of withdrawal from the open arm during the test (12). On the next day, 30 min after the injection of imipramine or saline, ETM avoidance was measured by recording the time taken for the rats to withdraw from the enclosed arm of the maze in three consecutive trials at 30-s intervals (baseline, avoidance 1 and 2). Following avoidance training (30 s), each animal was placed at the end of the same open arm used in the pre-exposure session and the time taken to leave this arm was recorded in three consecutive trials (escape 1 to 3 ), again with 30 -s inter-trial intervals. Immediately after the tests in the ETM, animals were placed in the center of the open field and allowed to freely explore for $5 \mathrm{~min}$.

\section{Statistical analysis}

A three-factor design was used to analyze the T-maze results, with the two treatments (treatment 1: sham x CORT; treatment 2: saline $x$ imipramine) as the independent factors and trials (baseline, avoidance 1 and 2 or escape 1, 2, and 3 latencies) as the dependent factors. Locomotor activity data in the open field were analyzed by two-way ANOVA. In case of significant interactions between factors, group comparisons were made by the Duncan test. A value of $\mathrm{P} \leq 0.05$ was considered to be significant.

\section{Results}

Figure 1 (upper panel) shows the effects of CORT and imipramine on ETM avoidance acquisition. Three-factor ANOVA showed a significant effect of trials $[F(2,58)$ $=27.15 ; \mathrm{P}<0.001]$, CORT $[\mathrm{F}(1,29)=5.10$; $P<0.05]$, imipramine $[F(1,29)=11.53 ; P<$ $0.001]$, and CORT by imipramine interaction $[F(1,29)=9.78 ; P<0.001]$. There was no trial by treatment interaction: trials by CORT $[F(1,29)=0.02 ; P>0.05]$, trials by imipramine $[F(1,29)=0.02 ; P>0.05]$, trials by CORT by imipramine $[F(1,29)=0.16 ; P>0.05]$. The Duncan post hoc test showed that animals treated with CORT were significantly different from all the other groups at baseline and in the two avoidance measurements, and that treatment with imipramine significantly counteracted the effects induced by CORT administration in the three trials.
The lower panel of Figure 1 shows the effects of CORT and imipramine on ETM escape. Three-factor ANOVA showed a significant effect of CORT $[F(1,29)=6.70 ; P<$ $0.05]$, imipramine $[F(1,29)=15.40 ; P<0.001]$, CORT by imipramine $[F(1,29)=9.84 ; P<0.01]$ and trials by imipramine $[F(2,58)=2.88 ; P<0.05]$. There was no significant effect of trials $[F(2,58)=1.09 ; P>0.05]$, trials by CORT $[F(2,58)$ $=2.88 ; P>0.05]$, or trials by CORT by imipramine $[F(2,58)$ $=0.09 ; \mathrm{P}>0.05]$. The Duncan post hoc test showed that sham animals treated with imipramine were different from the control group in all escape measurements, and that CORT counteracted the effects of imipramine in escape 2 and 3.

As shown in Figure 2, CORT did not alter the number of crossings $[F(1,29)=0.72 ; P>0.05]$ or the number of rearings $[F(1,29)=0.33 ; P>0.05]$. Imipramine altered both measurements: crossings $[F(1,29)=6.14 ; P<0.05]$ and
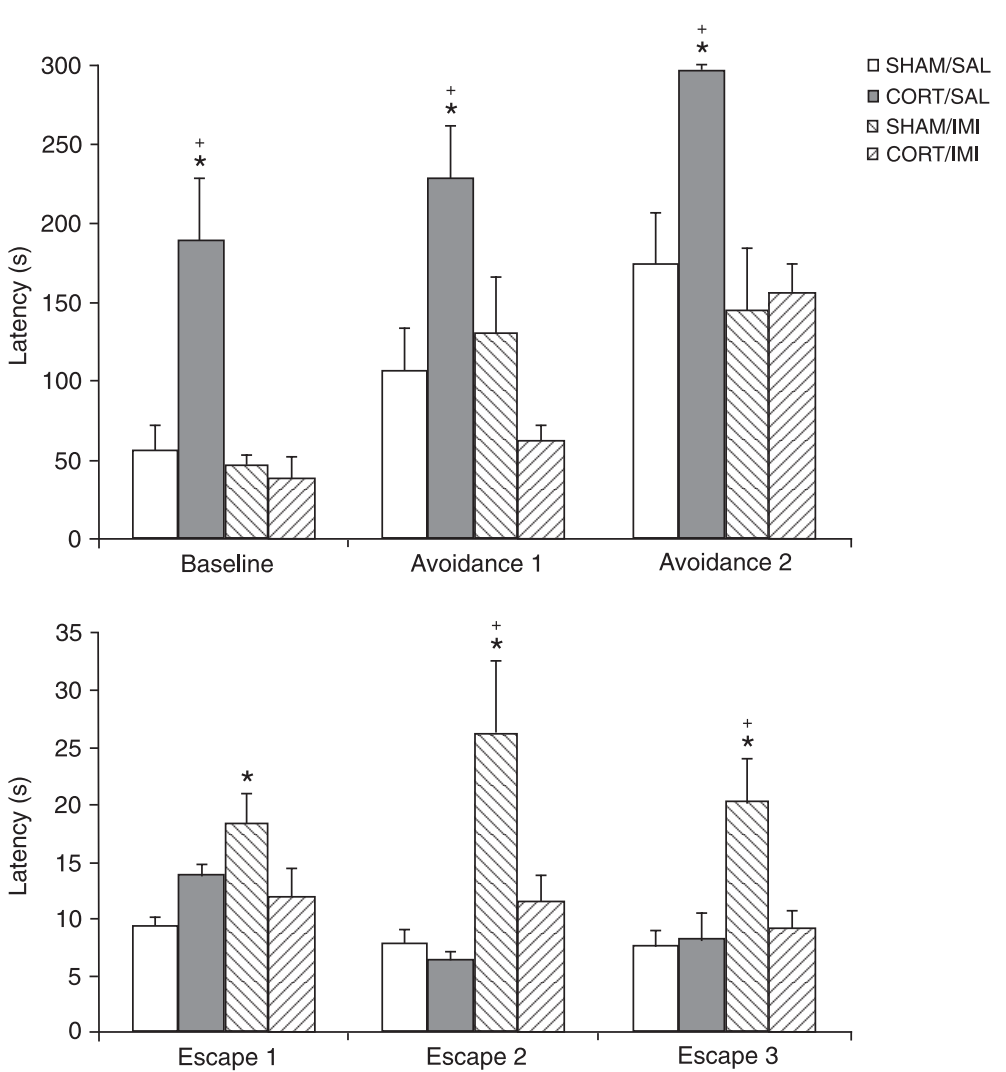

Figure 1. Effects of chronic corticosterone (200 mg, 21-day release pellets) and imipramine (15 mg/kg) on avoidance (upper panel) and escape (lower panel) latencies measured in the elevated T-maze. The latencies to leave the enclosed arm (baseline, avoidance 1 and avoidance 2) or one of the open arms (escape 1-3) were measured sequentially at 30 -s intervals beginning $30 \mathrm{~min}$ after ip treatment with imipramine or saline. Data are reported as means \pm SEM. $N=6-10$ per treatment group. $\mathrm{SAL}=$ saline; $\mathrm{CORT}=$ corticosterone; IMI $=$ imipramine. ${ }^{*} \mathrm{P}<0.05$ compared to control (SHAM/SAL) in the same trial; ${ }^{+} \mathrm{P}<0.05$ compared to the other groups in the same trial (ANOVA followed by the Duncan post hoc test). 
rearings $[F(1,29)=5.92 ; P<0.05]$. There were no significant interactions between treatments and crossings $[F(1,29)=0.06 ; P$ $>0.05]$ or treatments and rearings $[F(1,29)$ $=1.09 ; \mathrm{P}>0.05]$.

\section{Discussion}

The results of the present study suggest that chronic CORT facilitates ETM avoidance performance, an anxiogenic-like effect, without changing escape responses. Imipramine significantly reversed the anxiogenic effect of CORT, although by itself the drug did not alter avoidance measurements. On the other hand, imipramine inhibited escape responses, a panicolytic-like effect. This effect was also reversed by chronic treatment with CORT. Moreover, unlike chronic CORT, imipramine decreased both the number of crossings and rearings in an open field.

The anxiogenic effects observed on ETM avoidance corroborate previous evidence showing that treatment with CORT enhances acquisition and consolidation of a number of defense-related responses, such as inhibitory avoidance and contextual freezing $(1,2)$. Like ETM avoidance, these responses involve behavioral inhibition and occur in response to a potential threat. For the measurement of ETM avoidance, repeated exposure of the animal to the enclosed arm is necessary. When placed at the end of the enclosed arm for the first time, the rat does not see the open arms until it pokes its head beyond this arm walls. Being in the open arms seems to be an aversive experience, since rats have an innate fear of height and openness (13). Thus, in subsequent trials, the animal takes a longer time to withdraw from the enclosed arm towards the open space, showing the acquisition of ETM avoidance.

It is interesting to point out, however, that in the present study CORT also significantly enhanced baseline latencies. Traditionally, baseline latencies are a measurement of motor activity and not of anxiety (10). Nevertheless, since CORT did not alter motor behavior in the open field, the significant increase in baseline latencies observed might possibly reflect an anxious reaction to novelty. Indeed, it has been previously shown that longer-lasting hormonal stress responses induce increases in the reactions of the animals (i.e., movement activity, freezing, grooming) in a new environment (14). Also, activation of the hypothalamushypophysis-adrenal (HHA) axis seems to follow both reactions to novelty and to potential threat stimuli.

Imipramine efficiently counteracted the effects observed with CORT on ETM avoidance. It is interesting to point out that long-term administration of monoamine reuptake inhibitors has been found to affect neuronal plasticity in structures that modulate defense-related responses, such as the hippocampus (which presents high levels of glucocorticoid receptors), and to alter brain-derived neurotrophic factor levels (for a review, see Ref. 15). In fact, it was previously hypothesized that this could contribute to the therapeutic effects of these drugs (15). Further investigations are now being performed to determine if indeed imipramine alters neuronal plasticity induced by chronic CORT.

Nevertheless, imipramine by itself did not show any anxiolytic effects. These results are contrary to previous observations (10) obtained with the same dose range of the drug. However, and corroborating earlier evidence (10), imipramine also altered the number of crossings and rearings in the open field, thus showing a sedative action. Hence, it is possible that in the present case this decrease in motor activity masked the anxiolytic effects of the drug. On the other hand, it is important to point out that a study by our group (11) performed with two other reuptake inhibitors (clomipramine and fluoxetine) failed to show significant effects on ETM avoidance, although the drugs efficiently inhibited escape latencies, without altering locomotor activity. These results suggest that the escape measurement is more sensitive to treatment with antidepressant agents. The reason for this deserves to be better investigated.

Unlike avoidance measurements, the escape task was not significantly altered by treatment with chronic CORT. Escape is a defensive reaction performed in response to a proximal threat, in this case, direct exposure to an unconditioned aversive stimulus, i.e., open space. As previously mentioned, a study using the same dose of CORT and the same procedure adopted in the present investigation (implant of $200 \mathrm{mg} 21$-day release pellets), has shown that chronic CORT does not alter defensive behavior induced by another type of innate fear stimulus, i.e, exposure to a predator odor (4). As with ETM escape, in this particular study (4), animals were also placed in direct contact with the aversive stimulus (a chamber containing different con- 
centrations of trimethylthiazoline, a synthetic odor originally derived from fox feces).

In terms of psychopathology, ETM escape has been associated with panic disorder (8). Clinical observation has shown that although high plasma levels of cortisol accompany anticipatory anxiety and generalized anxiety disorder (16), no changes in the HHA axis or in cortisol levels are associated with panic attacks or panic disorder $(16,17)$. In a similar way, panicogenic stimuli (e.g., caffeine, sodium, lactate, $\mathrm{CO}_{2}$ ) can trigger panic attacks without a concomitant increase in cortisol release (for a review, see Ref. 16). On the basis of these observations, it has been proposed (16) that, in addition to the differences in symptomatology and pharmacological response, generalized anxiety and panic disorder may affect stress hormones differently. While anticipatory anxiety and generalized anxiety disorder activate both the HHA and the sympathoadrenal axes, a panic attack would be an emergency reaction that causes major sympathetic activation, but has little effect on the HHAaxis. Our results seem to corroborate this proposition.

Similar to what has been previously observed (10), treatment with imipramine inhibited escape responses, a panicolytic-like effect. On the other hand, chronic CORT was able to counteract the effects of imipramine. Since high levels of this adrenocorticotropic hormone have been associated with other clinical conditions (generalized anxiety, melancholic depression) but not with panic, these data appear to suggest that comorbidity interferes with the antipanic effect of the drug. In fact, comorbidity is frequently associated with poor compliance and poor response to antipanic agents (18).

Although our results show that chronic CORT does not alter ETM escape, a recent study (19) performed with another animal model of panic disorder, i.e., electrical stimulation of the dorsolateral periaqueductal grey, has demonstrated that the levels of plasma CORT were significantly increased after the induction of escape, a result that was altered by treatment with the selective serotonin reuptake inhibitor escitalopram. Nevertheless, since blood samples

\section{References}

1. Conrad CD, MacMillan DD, Tsekhanov S, Wright RL, Baran SE, Fuchs RA. Influence of chronic corticosterone and glucocorticoid receptor antagonism in the amygdala on fear conditioning. Neurobiol Learn Mem 2004; 81: 185-199.

2. McGaugh JL, Roozendaal B. Role of adrenal stress hormones in forming lasting memories in the brain. Curr Opin Neurobiol 2002; 12: 205-210.

3. Chrousos GP, Kino T. Glucocorticoid action networks and complex psychiatric and/or somatic disorders. Stress 2007; 10: 213-219.

4. Rosen JB, Donley MP, Gray D, West EA, Morgan MA, Schulkin J. Chronic corticosterone administration does not potentiate unconditioned freezing to the predator odor, were collected only 30 min after the escape reaction, it is possible that the increases in CORT levels were due to the blood sampling procedure adopted (tail clipping), and not a response to the activation of the panic circuit. Although it is a simple non-surgical blood collecting technique, tail clipping involves handling and restraining and may be a painful procedure. Animals that experienced a previous panic-like reaction are probably more susceptible to showing anticipatory anxiety in response to this aversive manipulation. Although the authors raise this concern, they do not further discuss it. To investigate this possibility, it would be important to use other blood sampling techniques, such as decapitation or indwelling catheters. Also, the fact that escitalopram decreased CORT levels is not a surprise. Like imipramine, escitalopram is not exclusively used for the treatment of panic, but also for generalized anxiety and depression. Indeed, it has been demonstrated that chronic treatment with these drugs decreases CORT levels and HHA axis activity (20).

In conclusion, the present results suggest that chronic treatment with CORT facilitates a behavioral defensive response that in clinical terms has been associated with generalized anxiety disorder. Chronic treatment with imipramine blocks this anxiogenic effect. These results confirm previous observations showing that the two responses measured by the ETM are in fact associated with distinct anxiety disorders $(8,10,11)$ and corroborate clinical data $(16,17)$ suggesting that generalized anxiety and panic disorder activate different neurobiological substrates, including stress hormones.

\section{Acknowledgments}

The authors are indebted to José Simões de Andrade for technical assistance (Universidade Federal de São Paulo, Santos). Research supported by FAPESP (\#2008/58815-1). M.B. Viana and B.B. dos Reis were recipients of research fellowships from CNPq. L. Diniz was the recipient of a fellowship grant from FAPESP (\#2009/50830-4). trimethylthiazoline. Behav Brain Res 2008; 194: 32-38.

5. Blanchard DC, Griebel G, Blanchard RJ. Mouse defensive behaviors: pharmacological and behavioral assays for anxiety and panic. Neurosci Biobehav Rev 2001; 25: 205-218.

6. Gray JA, McNaughton N. The neuropsychology of anxiety: an enquiry into the functions of the septo-hippocampal system. Oxford: Oxford University Press; 2000.

7. McNaughton N, Corr PJ. A two-dimensional neuropsychology of defense: fear/anxiety and defensive distance. Neurosci Biobehav Rev 2004; 28: 285-305.

8. Viana MB, Tomaz C, Graeff FG. The elevated T-maze: a new animal model of anxiety and memory. Pharmacol Biochem Behav 1994; 49: 549-554. 
9. Graeff FG, Netto CF, Zangrossi H Jr. The elevated T-maze as an experimental model of anxiety. Neurosci Biobehav Rev 1998; 23: 237-246.

10. Teixeira RC, Zangrossi H, Graeff FG. Behavioral effects of acute and chronic imipramine in the elevated T-maze model of anxiety. Pharmacol Biochem Behav 2000; 65: 571-576.

11. Poltronieri SC, Zangrossi H Jr, de Barros Viana M. Antipaniclike effect of serotonin reuptake inhibitors in the elevated T-maze. Behav Brain Res 2003; 147: 185-192.

12. Sena LM, Bueno C, Pobbe RL, Andrade TG, Zangrossi H $\mathrm{Jr}$, Viana MB. The dorsal raphe nucleus exerts opposed control on generalized anxiety and panic-related defensive responses in rats. Behav Brain Res 2003; 142: 125-133.

13. Graeff FG, Zangrossi H Jr. Animal models of anxiety. In: D'Haenen D, Den Boer JA, Willner P (Editors), Biological Psychiatry. London: John Wiley \& Sons; 2002. p 1-15.

14. Pivina SG, Akulova VK, Ordyan NE. Characteristics of behavior and stress reactivity of the hypophyseal-adrenocortical system in rats with prenatal inhibition of testosterone metabolism. Neurosci Behav Physiol 2007; 37: 53-58.

15. Hodes GE, Hill-Smith TE, Suckow RF, Cooper TB, Lucki I.
Sex-specific effects of chronic fluoxetine treatment on neuroplasticity and pharmacokinetics in mice. J Pharmacol Exp Ther 2010; 332: 266-273.

16. Graeff FG. Anxiety, panic and the hypothalamic-pituitaryadrenal axis. Rev Bras Psiquiatr 2007; 29 (Suppl 1): S3-S6.

17. Garcia-Leal C, Parente AC, Del-Ben CM, Guimaraes FS, Moreira AC, Elias LL, et al. Anxiety and salivary cortisol in symptomatic and nonsymptomatic panic patients and healthy volunteers performing simulated public speaking. Psychiatry Res 2005; 133: 239-252.

18. Marchesi C. Pharmacological management of panic disorder. Neuropsychiatr Dis Treat 2008; 4: 93-106.

19. Lim LW, Blokland A, van Duinen M, Visser-Vandewalle V, Tan S, Vlamings R, et al. Increased plasma corticosterone levels after periaqueductal gray stimulation-induced escape reaction or panic attacks in rats. Behav Brain Res 2011; 218 : 301-307.

20. Michelson D, Galliven E, Hill L, Demitrack M, Chrousos G, Gold P. Chronic imipramine is associated with diminished hypothalamic-pituitary-adrenal axis responsivity in healthy humans. J Clin Endocrinol Metab 1997; 82: 2601-2606. 